\title{
Evaluation of Travelling Vortex Speed by Means of Dynamic Mode Decomposition and Residual Vorticity
}

\author{
Tomáš Hyhlík ${ }^{1, a}$ and Hana Netřebská ${ }^{1}$ \\ CTU in Prague, FME, Department of Fluid Dynamics and Thermodynamics, Technická 4, 16607 Prague
}

\begin{abstract}
The article deals with the analysis of synthetic jet flow field by means of dynamic mode decomposition (DMD) method. The speed of travelling vortex ring which is connected with synthetic jet creation is evaluated using the wavelength identified from DMD modes. Vortices are identified in the DMD modes by using residual vorticity which allows to identify regions in the flow field where fluid particles perform rotational motion. The analysis is based on the data from the numerical simulation of synthetic jet into quiescent air by using ANSYS Fluent code. The regime of synthetic jet with $\mathrm{Re}=329$ and Stk $=19.7$ is chosen. An increase in the vortex speed close to the orifice and then the decrease is observed with maximum reaching almost one and half of averaged blowing orifice centerline velocity.
\end{abstract}

\section{Introduction}

Current work is dealing with the analysis of unsteady flow field with travelling vortex ring and from the point of view of modal decomposition is continuation of e.g. the works $[1,2]$. From the point of view of analysis of synthetic jet flow it is indirect visualization method unlike the works $[3,4]$, where is the subject of synthetic jet flow studied by using direct visualization.

The visualization of vortex structures in the DMD modes is problematic. There is a standard approach based on vector lines, but identification of vortex core is inaccurate. The aim is to refine identification of vortices in the DMD modes by using the concept of residual vorticity.

\section{Parameters of Synthetic Jet}

Velocity scale is typically defined as time averaged blowing orifice centerline velocity over an entire cycle

$$
U_{0}=\frac{1}{T} \int_{0}^{T_{E}} u_{0}(r=0, t) \mathrm{d} t,
$$

where $T_{E}$ is blowing time and $T=1 / f$ is oscillation period. Reynolds number can be defined using time averaged blowing orifice centerline velocity $U_{0}$ as

$$
\operatorname{Re}=\frac{2 U_{0} D}{v}
$$

where $D$ is orifice diameter. Stokes number is defined as

$$
\mathrm{Stk}=D \sqrt{\frac{2 \pi f}{v}}
$$

where $f$ is frequency and $v$ is kinematic viscosity. The regime of synthetic jet with $\mathrm{Re}=329$ and $\mathrm{Stk}=19.7$ is chosen.

\footnotetext{
a e-mail: tomas.hyhlik@fs.cvut.cz
}

\section{Numerical Simulation}

Unsteady incompressible laminar flow simulation has been performed using commercial solver ANSYS Fluent. The flow is assumed to be axisymmetric and is simulated on rectilinear grid with 81550 cells using non iterative time advancement method with second order implicit scheme. Fractional step scheme is used for pressure velocity coupling. Convective terms are discretized using third order MUSCL scheme. Both the flow in the orifice and actuator cavity are included in the simulation to get more accurate results. The effect of oscillating diaphragm is replaced by velocity boundary condition which should guarantee achievement of required Reynolds number

$$
u(t)=\frac{\pi}{2} \frac{\operatorname{Re} \eta}{\rho D} \frac{D^{2}}{D_{L}^{2}} \sin (2 \pi f t),
$$

where $\eta$ is dynamic viscosity, $\rho$ is air density and $D_{L}$ is inlet diameter i.e. diameter of oscillating diaphragm. Details of the numerical simulation can be found in reference [5].

\section{Dynamic Mode Decomposition}

Dynamic mode decomposition is a kind of modal decomposition method which was developed based on Koopman analysis of dynamical system [6]. DMD algorithm approximates Koopman modes from finite set of data. This section is mainly based on the reference [7]. Let the velocity field be represented by ensemble of snapshots sampling at interval $\Delta t$ with a form of matrix $\mathbf{V}_{1}^{N}=\left\{\mathbf{v}_{1}, \mathbf{v}_{2}, \ldots, \mathbf{v}_{N}\right\}$, where the column vector $\mathbf{v}_{j}$ contains velocity field at $j$ th time step. Let's consider linear mapping A that maps each snapshot to the next one in the following way

$$
\mathbf{v}_{j+1}=\mathbf{A} \mathbf{v}_{j}
$$

This mapping is assumed to be same over the full sampling interval $[0,(N-1) \Delta t]$. If the flow fields stem from 
a nonlinear process, this assumption corresponds to linear tangent approximation [7]. The assumption of a constant mapping between the snapshots $\mathbf{v}_{j}$ allows us to formulate the sequence of flow fields as a Krylov sequence

$$
\mathbf{V}_{1}^{N}=\left\{\mathbf{v}_{1}, \mathbf{A} \mathbf{v}_{1}, \mathbf{A}^{2} \mathbf{v}_{1}, \ldots, \mathbf{A}^{N-1} \mathbf{v}_{1}\right\}
$$

The goal of DMD is the extraction of dynamic characteristics such as eigenvalues and eigenvectors which are described by linear operator $\mathbf{A}$ based on sequence $\mathbf{V}_{1}^{N}$. Supposing that it is possible to write last snapshot $\mathbf{v}_{N}$ as linear combination of previous $N-1$ snapshots, for sufficiently long sequence of the snapshots

$$
\mathbf{v}_{N}=a_{1} \mathbf{v}_{1}+a_{2} \mathbf{v}_{2}+\ldots+a_{N-1} \mathbf{v}_{N-1}+\mathbf{r}
$$

where $\mathbf{a}^{T}=\left\{a_{1}, a_{2}, \ldots, a_{N-1}\right\}$ and $\mathbf{r}$ is residual vector. It is possible to write following relations

$$
\begin{gathered}
\mathbf{A}\left\{\mathbf{v}_{1}, \mathbf{v}_{2}, \mathbf{v}_{3}, \ldots, \mathbf{v}_{N-1}\right\}=\left\{\mathbf{v}_{2}, \mathbf{v}_{3}, \mathbf{v}_{4}, \ldots, \mathbf{v}_{N}\right\} \\
\left\{\mathbf{v}_{2}, \mathbf{v}_{3}, \mathbf{v}_{4}, \ldots, \mathbf{v}_{N}\right\}=\left\{\mathbf{v}_{2}, \mathbf{v}_{3}, \mathbf{v}_{4}, \ldots, \mathbf{V}_{1}^{N-1} \mathbf{a}\right\}+\mathbf{r e}_{N-1}^{T},
\end{gathered}
$$

or in matrix form

$$
\mathbf{A} \mathbf{V}_{1}^{N-1}=\mathbf{V}_{2}^{N}=\mathbf{V}_{1}^{N-1} \mathbf{Z}+\mathbf{r e}_{N-1}^{T}
$$

where $\mathbf{e}_{N-1}^{T}$ is unit vector of dimension $N-1$. Matrix $\mathbf{Z}$ is companion matrix

$$
\mathbf{Z}=\left(\begin{array}{ccccc}
0 & & & & a_{1} \\
1 & 0 & & & a_{2} \\
& \ddots & \ddots & & \vdots \\
& & 1 & 0 & a_{N-2} \\
& & & 1 & a_{N-1}
\end{array}\right) .
$$

The eigenvalues of $\mathbf{Z}$ are approximations of eigenvalues of linear operator A. Decomposition based on companion matrix $\mathbf{Z}$ is mathematically correct, but more robust algorithm based on similar matrix $\tilde{\mathbf{Z}}$ is used in this work. Robustness is achieved by preprocessing step using singular value decomposition of data sequence [7] $\mathbf{V}_{1}^{N-1}=\mathbf{U} \mathbf{\Sigma} \mathbf{W}^{H}$. Substituting singular value decomposition into (10) and rearranging the resulting expression we have

$$
\mathbf{U}^{H} \mathbf{A} \mathbf{U}=\mathbf{U}^{H} \mathbf{V}_{2}^{N} \mathbf{W} \boldsymbol{\Sigma}^{-1}=\tilde{\mathbf{Z}} .
$$

Dynamic modes can be calculated using equation [7]

$$
\boldsymbol{\Phi}_{i}=\mathbf{U y}_{\mathbf{i}}
$$

where $\mathbf{y}_{i}$ is $i$ th eigenvector of $\tilde{\mathbf{Z}}$

$$
\tilde{\mathbf{Z}} \mathbf{y}_{i}=\mu_{i} \mathbf{y}_{i}
$$

Eigenvalues $\mu_{i}$ approximate eigenvalues of full mapping matrix $\mathbf{A}$ and provide temporal dynamics of the flow field.

\section{Vortex Identification}

The work [8] is offering an impressive concept of triple decomposition of motion where the tensor of velocity gradient is splitted into three parts. The first part is representing the elongation of fluid portion, the second part is connected with rigid body rotation and the third part is effective pure shearing tensor. The reference [8] presents both the simple two dimensional decomposition and the general three dimensional approach. The aim of this section is to extend the two dimensional approach to be applicable to axisymmetric flow. The key idea of the mentioned work is to rotate the coordinate system to the so called basic reference frame where it is possible to clearly recognize the kind of fluid motion.

It is possible to express the continuum particle speed $u_{i}$ when it changes its position from point $x_{j}$ to point $x_{j}+\mathrm{d} x_{j}$ as

$$
u_{i}\left(x_{j}+\mathrm{d} x_{j}\right)=u_{i}\left(x_{j}\right)+S_{i j} \mathrm{~d} x_{j}+\Omega_{i j} \mathrm{~d} x_{j},
$$

where $S_{i j}$ is symmetrical part of velocity gradient tensor and $\Omega_{i j}$ is antisymmetric part of velocity gradient tensor. Symmetrical part is

$$
\mathbf{S}=\left(\begin{array}{ccc}
\frac{\partial v_{r}}{\partial r} & 0 & \frac{1}{2}\left(\frac{\partial v_{r}}{\partial z}+\frac{\partial v_{z}}{\partial r}\right) \\
0 & \frac{v_{r}}{r} & 0 \\
\frac{1}{2}\left(\frac{\partial v_{r}}{\partial z}+\frac{\partial v_{z}}{\partial r}\right) & 0 & \frac{\partial v_{z}}{\partial z}
\end{array}\right)
$$

in the case of axisymmetric flow with zero swirl velocity and antisymmetric part can be simplified as

$$
\boldsymbol{\Omega}=\left(\begin{array}{ccc}
0 & 0 & \frac{1}{2}\left(\frac{\partial v_{r}}{\partial z}-\frac{\partial v_{z}}{\partial r}\right) \\
0 & 0 & 0 \\
-\frac{1}{2}\left(\frac{\partial v_{r}}{\partial z}-\frac{\partial v_{z}}{\partial r}\right) & 0 & 0
\end{array}\right) .
$$

The tensor in Cartesian coordinates can be converted from cylindrical coordinates using

$$
\left(\begin{array}{lll}
S_{x x} & S_{x y} & S_{x z} \\
S_{y x} & S_{y y} & S_{y z} \\
S_{z x} & S_{z y} & S_{z z}
\end{array}\right)=\mathbf{B}\left(\begin{array}{lll}
S_{r r} & S_{r \theta} & S_{r z} \\
S_{\theta r} & S_{\theta \theta} & S_{\theta z} \\
S_{z r} & S_{z \theta} & S_{z z}
\end{array}\right) \mathbf{C},
$$

where transformation matrices are

$$
\mathbf{B}=\left(\begin{array}{ccc}
\cos \theta & -\sin \theta & 0 \\
\sin \theta & \cos \theta & 0 \\
0 & 0 & 1
\end{array}\right), \mathbf{C}=\left(\begin{array}{ccc}
\cos \theta & \sin \theta & 0 \\
-\sin \theta & \cos \theta & 0 \\
0 & 0 & 1
\end{array}\right) \text {. }
$$

The angle $\theta$ is arbitrary in the case of axisymmetric flow. Let angle be $\theta=\pi / 2$. We get

$$
\mathbf{S}=\left(\begin{array}{lll}
S_{x x} & S_{x y} & S_{x z} \\
S_{y x} & S_{y y} & S_{y z} \\
S_{z x} & S_{z y} & S_{z z}
\end{array}\right)=\left(\begin{array}{ccc}
S_{\theta \theta} & 0 & 0 \\
0 & S_{r r} & S_{r z} \\
0 & S_{r z} & S_{z z}
\end{array}\right)
$$

We have to find principal values and principal axes of the matrix of kind

$$
\left(\begin{array}{ccc}
S_{x x} & S_{x y} & 0 \\
S_{y x} & S_{y y} & 0 \\
0 & 0 & S_{z z}
\end{array}\right)
$$

The value of $S_{z z} \neq 0$ is in general nonzero in the case of axisymmetric flow. Eigenvalues of matrix (21) are

$$
\lambda_{1}=e_{1}+s, \lambda_{2}=e_{2}-s, \lambda_{3}=e_{3},
$$

where

$$
s=\frac{1}{2} \sqrt{\left(S_{x x}-S_{y y}\right)^{2}+4 S_{x y}^{2}}
$$

and

$$
e_{1,2}=e=\frac{1}{2}\left(S_{x x}+S_{y y}\right), e_{3}=S_{z z}
$$




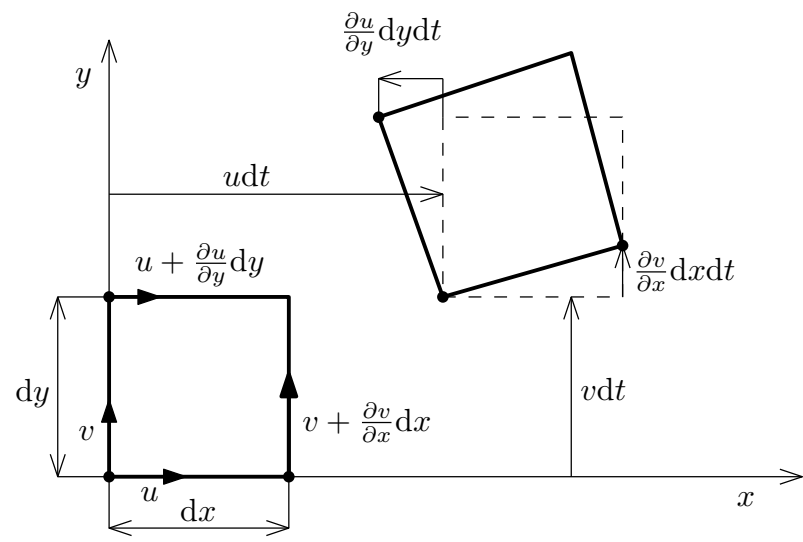

Fig. 1. Predominant rotation; the case where $\frac{\partial u}{\partial y}<0$ and $\frac{\partial v}{\partial x}>0$.

The velocity gradient tensor can be in principal axes expressed as

$$
\nabla \mathbf{v}=\left(\begin{array}{lll}
e & 0 & 0 \\
0 & e & 0 \\
0 & 0 & e_{3}
\end{array}\right)+\left(\begin{array}{ccc}
s & 0 & 0 \\
0 & -s & 0 \\
0 & 0 & 0
\end{array}\right)+\left(\begin{array}{ccc}
0 & -\omega & 0 \\
\omega & 0 & 0 \\
0 & 0 & 0
\end{array}\right)
$$

where

$$
\omega=\frac{1}{2}\left(\frac{\partial v_{y}}{\partial x}-\frac{\partial v_{x}}{\partial y}\right)
$$

An arbitrary rotation around $z$ axis does not change the first and the third matrix in the relation (25). The first tensor in the relation (25) has no influence on shear deformation. It is possible to take into consideration only the second and the third part and rotate around $z$ axis about angle $\alpha=\frac{3}{4} \pi$

$$
\nabla \mathbf{v} \rightarrow \mathbf{D}\left(\begin{array}{ccc}
s-\omega & 0 \\
\omega & -s & 0 \\
0 & 0 & 0
\end{array}\right) \mathbf{F}=\left(\begin{array}{ccc}
0 & s-\omega & 0 \\
s+\omega & 0 & 0 \\
0 & 0 & 0
\end{array}\right)
$$

where transformation matrix $\mathbf{D}$ is

$$
\mathbf{D}=\left(\begin{array}{ccc}
\cos \alpha & \sin \alpha & 0 \\
-\sin \alpha & \cos \alpha & 0 \\
0 & 0 & 1
\end{array}\right)=\left(\begin{array}{ccc}
-\frac{\sqrt{2}}{2} & \frac{\sqrt{2}}{2} & 0 \\
-\frac{\sqrt{2}}{2} & -\frac{\sqrt{2}}{2} & 0 \\
0 & 0 & 1
\end{array}\right)
$$

and transformation matrix $\mathbf{F}$ is

$$
\mathbf{F}=\left(\begin{array}{ccc}
\cos \alpha & -\sin \alpha & 0 \\
\sin \alpha & \cos \alpha & 0 \\
0 & 0 & 1
\end{array}\right)=\left(\begin{array}{ccc}
-\frac{\sqrt{2}}{2} & -\frac{\sqrt{2}}{2} & 0 \\
\frac{\sqrt{2}}{2} & -\frac{\sqrt{2}}{2} & 0 \\
0 & 0 & 1
\end{array}\right)
$$

Velocity gradient tensor is after transformation in so called basic reference frame [8]. It is possible to examine kinematics of the flow in the basic reference frame clearly.

All of us intuitively know that vortex is somehow connected with vorticity but on the other hand we know that there are cases like laminar shear flow where the value of vorticity is nonzero and there is not any vortex. The work [8] defines vortex as the case where the value of vorticity predominates the shear $|\omega| \geq|s|$. Let's assume only shear and vorticity parts of the velocity gradient tensor, then it has only two nonzero components in the basic reference frame $\frac{\partial u}{\partial y}$ and $\frac{\partial v}{\partial x}$ having opposite sign in the case of predominant vorticity, see figure 1 . The residual shear parameter is in this case $s_{R E S}=0$ and shear parameter is $s_{S H}=s$.

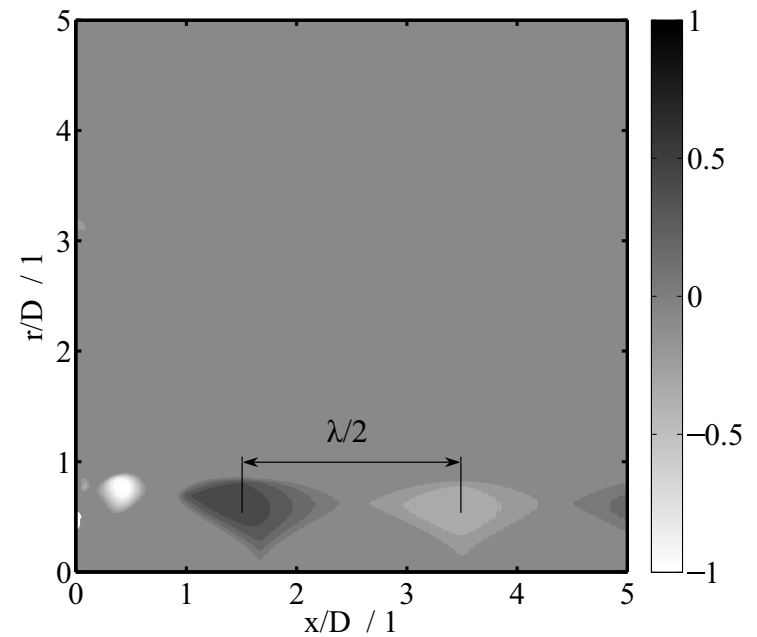

Fig. 2. Residual vorticity contours of imaginary part of the first DMD mode which is connected with frequency $f_{1}=f$

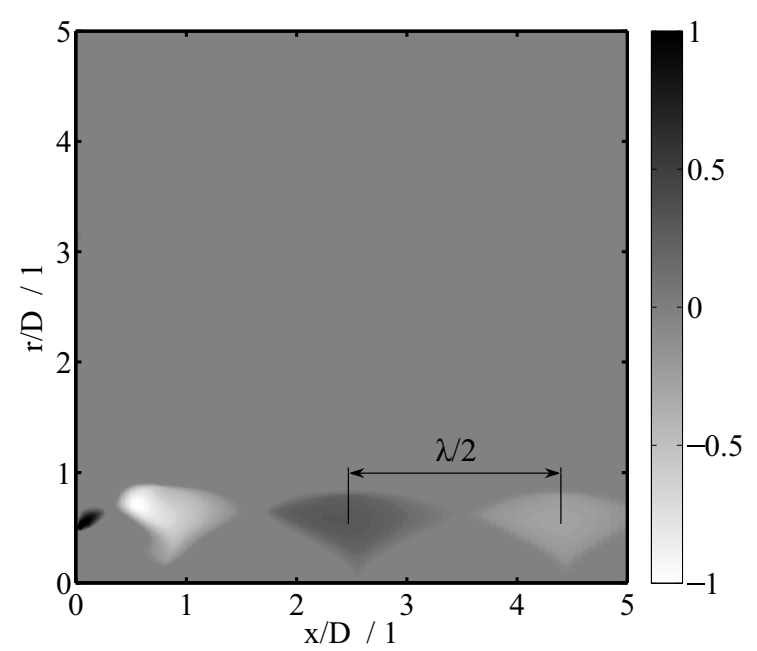

Fig. 3. Residual vorticity contours of real part of the first DMD mode which is connected with frequency $f_{1}=f$

Let the residual vorticity be

$$
\omega_{R E S}=\operatorname{sgn}(\omega)[|\omega|-|s|]
$$

and shear vorticity be

$$
\omega_{S H}=\operatorname{sgn}(\omega)|s| .
$$

Examples of residual vorticity distributions in the DMD modes are depicted in the figures 2, 3, 4 and 5.

\section{Results}

There are basically two types of behaviour connected with spatial DMD modes. The first one is the case where the real part dominates over imaginary part. This kind of behaviour in the dynamic mode can indicate travelling wave like structure in the flow field [9]. The second kind of behaviour where the real and imaginary parts are similar, but there is space shift in the flow field. The space shift is typically one quarter of wave length which can be measured 


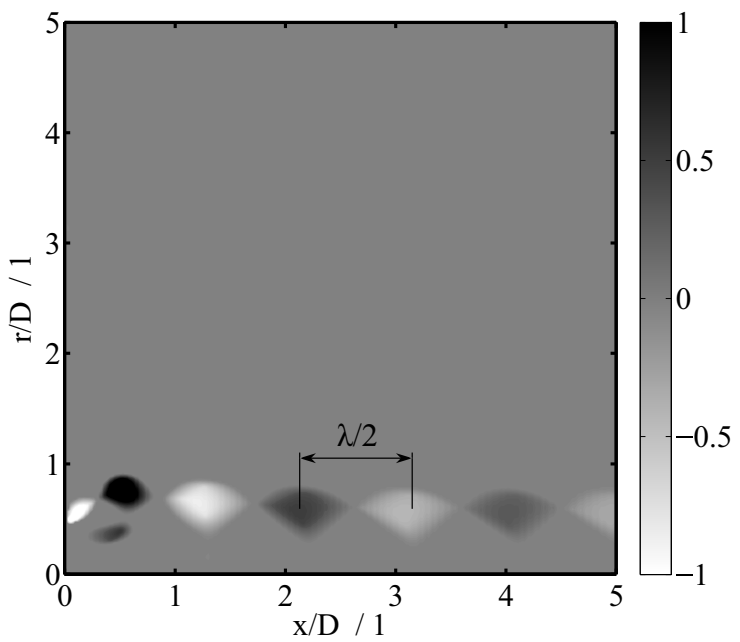

Fig. 4. Residual vorticity contours of imaginary part of the second DMD mode which is connected with frequency $f_{2}=2 f$

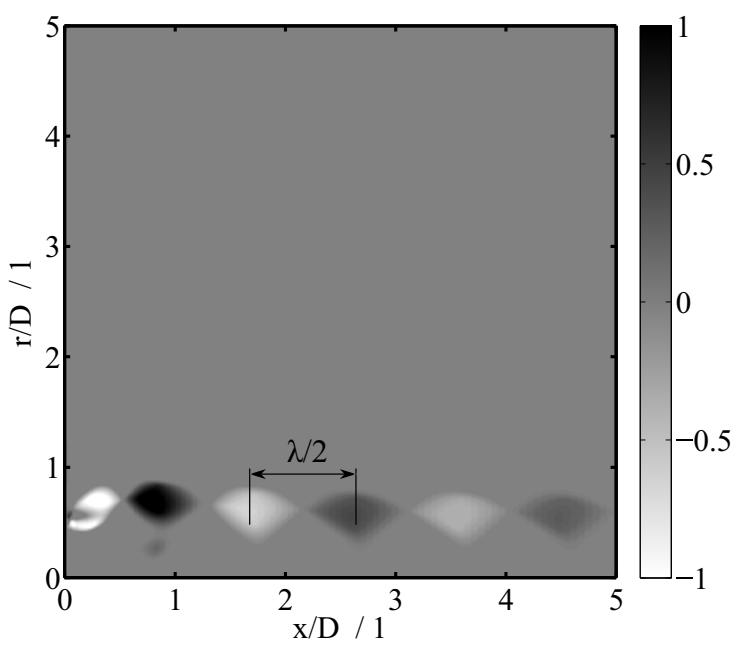

Fig. 5. Residual vorticity contours of real part of the second DMD mode which is connected with frequency $f_{2}=2 f$

from the modes. This kind of behaviour is typical for the travelling vortex structures and is observed in the figures 2 , 3,4 and 5. There is a travelling vortex ring in the flow field depicted in the mentioned figures. The vortex ring is travelling from left to right in the $x$ direction, where $x=0$ corresponds to orifice exit. It is possible to observe opposite vortex rotation in the DMD modes in the figures, where vortices are visualized using residual vorticity. It is possible to directly measure wavelength $\lambda$ in the case of every single DMD mode. The wavelength is changing with spatial position. The vortex speed is connected with wavelength as

$$
u_{c}=\lambda f_{m},
$$

where $f_{m}$ is frequency connected with given mode. There is an increase in the vortex speed close to the orifice and then is the vortex speed decreasing as shown in the figure 6 . The maximum vortex speed is reaching almost one and half of averaged blowing orifice centerline velocity over an entire cycle $U_{0}$ in this case. The predicted behaviour is consistent with reference [10] but predicted maximal vortex speed is slightly higher.

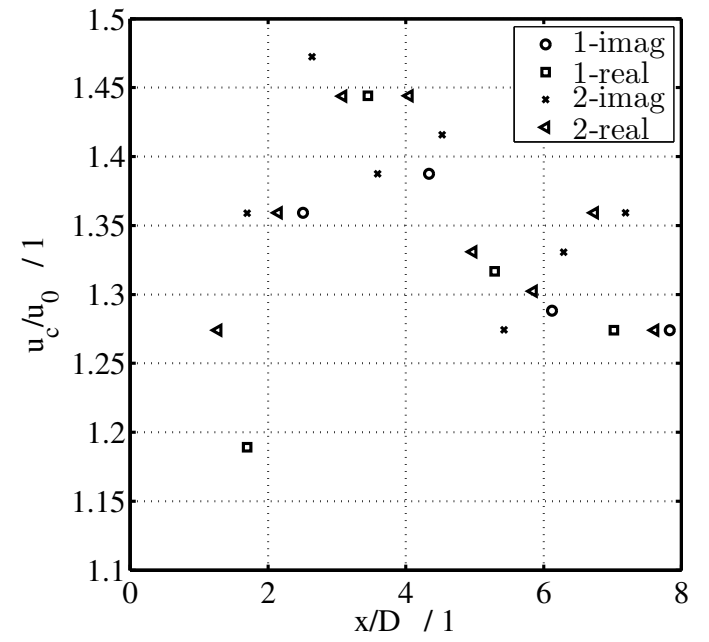

Fig. 6. Vortex speed normalized by averaged blowing orifice centerline velocity

\section{Conclusions}

The speed of travelling vortex ring is evaluated by using DMD modes, where the vortices are identified using residual vorticity. The vortex structure identification based on residual vorticity is modified to be applicable to axisymmetric flow. The contours of residual vorticity of individual modes are used to measure spatial change of wave length which together with the mode frequency allows to calculate speed of travelling vortex.

\section{Acknowledgement}

The support from Czech Science Foundation under Grant No. 14-08888S is gratefully acknowledged.

\section{References}

1. T. Hyhlík, P. Železný, J. Č́ížek, EPJ Web of Conferences, 45, (2013), 01043

2. T. Hyhlík, H. Netřebská, EPJ Web of Conferences, 92, (2015), 02026

3. Z. Broučková, Z. Trávníček, P. Šafařík, EPJ Web of Conferences, 25, (2012), 01007

4. Z. Trávníček, Z. Broučková, J. Kordík, T. Vít, Journal of Visualization, (in press; online available March 2015)

5. T. Hyhlík, H. Netřebská, 33th Conference of Departments of Fluid Mechanics and Thermomechanics, (Prague 2014)

6. C. W. Rowley, I. Mezić, S. Bagheri, P. Schlatter, D. S. Henningson, J. Fluid Mech., 641, (2009), 115-127.

7. P. J. Schmid, J. Fluid Mech., 656, (2010) 5-28.

8. V. Kolár̆, International Journal of Heat and Fluid Flow, 28, (2007), 638-652.

9. O. Frederich, D. M. Luchtenburg, Seventh International Symposium on Turbulence and Shear Flow Phenomena, (Ottawa 2011)

10. B. L. Smith, A. Glezer, Physics of Fluids, 10(9), (1998), 2281-2297 